\title{
Etude comparative des turbines Kaplan et Dériaz
}

\section{A comparative study of the Kaplan and Dériaz turbines}

\author{
PAR P. DERIAZ

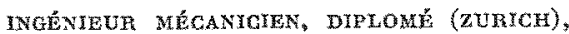

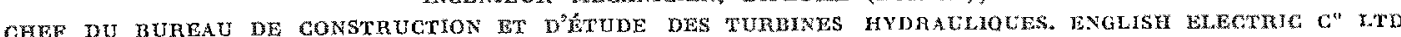

\begin{abstract}
Bref résumé historique du développement de la turbine Dériaz et de ses applications.

Considérations hydrauliques et mécaniyues comparant la tubine Kaplan à la turbine Dériaz.

Awantages de cete derniere en anétioration du rendement; en reduction du facteur de cavitrtion; en reduction des dimerstons de la centruls et de la capacité du pont; an reduction de la nesse demballement conduisant it une gén ratrice plus économique; en reduction des accrobssments de debit en survitesse conduisant à une économie d'excavation du turnel aval, pour une centrale sonterraine.

Résultats d'esals comparatifs en modele rédxit équipé allernativement avec roue baplan et avec roue Deriaz dars la mêtne biane spirale, mêmes dispositifs distributear et aspirateur, et construites pour des conditions identiques doperation.
\end{abstract}

Le présent mémoire a été présenté pour la première lois à l'occasion des fêtes du «ubilć d'Or $*$ de l'Indian Institute of Science, à Bangalore, en novembre 1959.

\section{蜘}

La turbine Dériaz a été réalisée à la suite d'études et de travaux de mise au point ayant pour objet de créer une turbine fonctionnant zus des chutes de plus de 60 mètres et possédant une caracléristique de rendemenl/charge d'allure 《aplatie $»$ tout en présentant la stabllité de fonctionnement aux ouvertures partielles des Kaplan classiques. On sait que l'utilisation d'une turbine Kaplan devient extrêmement diffcile à des chutes au-delà de 60 mètres, tant du
Brief review of historical development of the Deriaz turbine and its applications.

Hydraulic and mechanical consiticrations when comparing the Kaptan and the Deriuz lurbines. Advantages of the latter in incredsed effoiency, in reduction of cavitation factor, in reduclion of size of power house and sizs of crane, it reduction of runaway speed which leads to a more economical generator, and in reduction of excessive flow at overspeeds, leuding to reduction in tailrace tunnel excavation for an underground station.

Comparative test results on a model turbine fitted with a Kaplan runner and alternatiocly with a Deriaz runner in identical spiral casing, guide apporatus and draft hobe and designed for the same operating conditions. point de vue hydralique que mécanique; ce problème a été résolu a moyen d'une roue â tcoulement mixte et a pales au pas variable. L'historique de ce travail a été publié [1].

Celte nouvelle réalisation fut mise en exploim tation pour la premiere fois h la station de pomm page et de production d'énergie Sir Adam Beck, Niagara, de l'Hydro-Electric Power Commission of Ontario, dans laquelle les groupes doivent fonctionner tant comme pompes que comme turbines. La centrale comporte 6 groupes avec une capacite de pompage globale de $330000 \mathrm{HP}$ sous une charge de $27,50 \mathrm{~m}$ (voir fig. 1 et 2). Ces groupes sont en exploitation depuis juillet 1957 , fonctionnant suivant des programmes hebdomadaires complets, avec des inversions de fonc- 


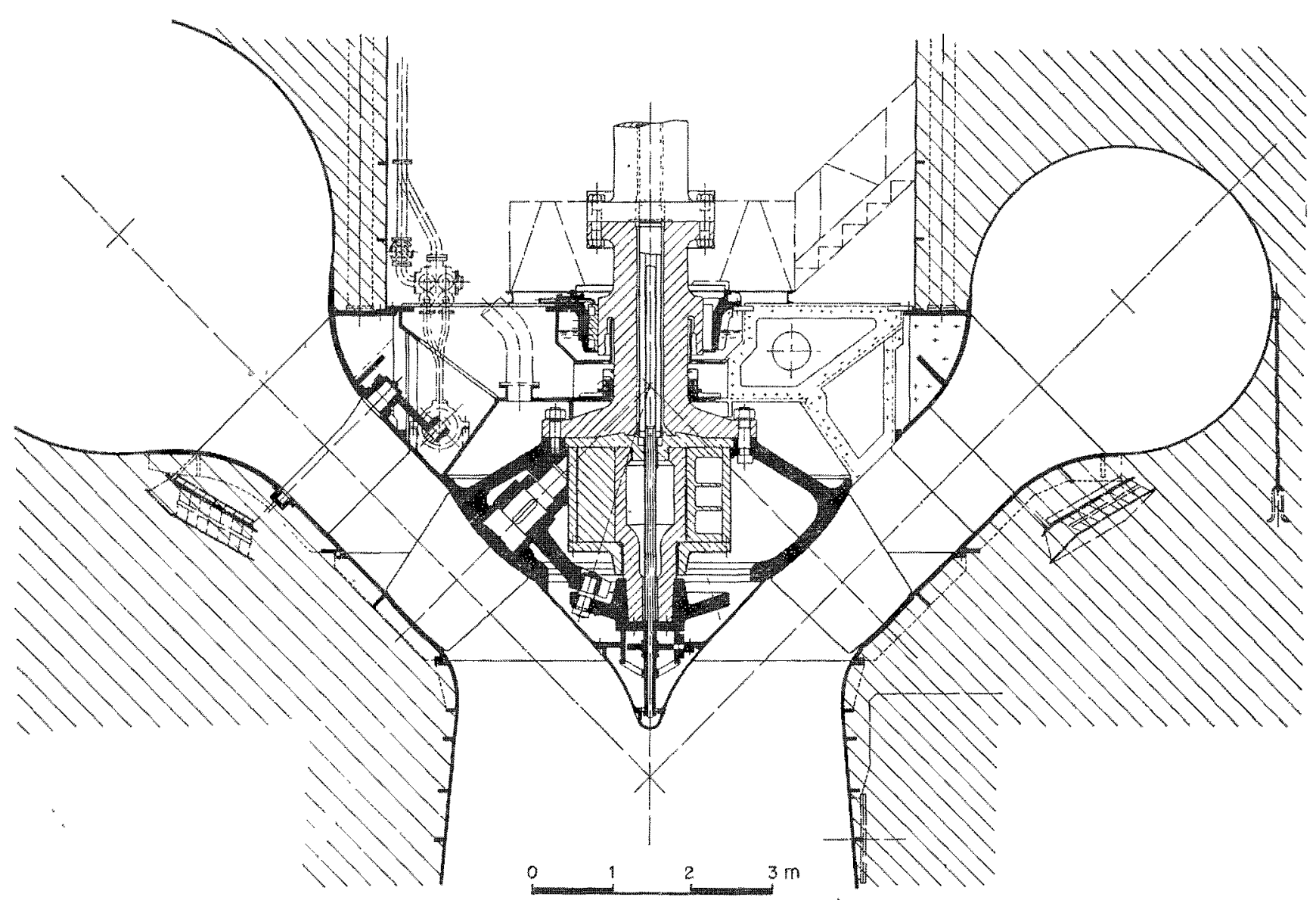

Fia. 1. - Pompes-turbines réversibles, pour la centrale de "Sir Adam Beck" Niagara, station de pompage et de production d'énergie de l'Hydro-Electric Power Commission of Ontario.

Puissance de la tarbine : 46000 B.HP. - Puissance d'entrée de la pompe : 52500 B.HP. Vitesse: $92,4 \mathrm{tr} / \mathrm{mn}$ - Chute 12 à 27,50 metres.

tionnement chaque jour et chaque nuit. Trois turbines Dériar, d'une puissance unitaire de $110000 \mathrm{HP}$, sont actuellement en construction pour une centrale espagnole. Ces machines sont réversibles, p o u a n $t$ également fonctionner comme pompes, sous une charge maximale de $73 \mathrm{~m}$ (voir fig. 3).

D'une étude détallée, l'auteur a pu conclure que, pour des chutes dépassant $36 \mathrm{~m}$, les turbines Dériaz à écoulement mixte présentent des avantages marqués sur la turbine Kaplan à écoulement axial. Poux des chutes inférieures à $36 \mathrm{~m}$, la vitesse supérieure de la Kaplan et, par suite, la génératrice à meilleur marché, mililent en faveur de celle dernière.

Afin de démontrer les avantages de la turbine Dériaz, le reste du présent article présente les résultats de l'étude effectuée récemment, dans laquelle fut comparce une turbine Dériaz dune turbine Kaplan, pour une installation donnée. Les caractéristiques d'étude des deux machines sont. les mêmes:
- Puissance: 30000 B.HP.

- Chute: $55 \mathrm{~m}$.

- Vitesse: $300 \mathrm{tr} / \mathrm{mn}$.

- Vitesse spécifique: $n_{\mathrm{s}}=350$ (unités métriques).

Les coupes transversales des deux turbines sont montrées dans les figures 4 et $5 ;$ on voit que le diamètre du cercle de vannage (1) est le même dans les deux cas, bien que la hauteur des direchices soit légèrement plus grande dans le cas de la Kaplan (2).

Dans le cas particulier, les axes des pales de la Dériaz sont inclinés à $30^{\circ}$ par rapport à l'horizontale, alors que l'angle de ces axes adopté pour Niagara élait de $45^{\circ}$ (fig. 1). Il a été nécessaire de changer cet angle afin de l'adapter à la vitesse spécifique plus élevée de la turbine considérée dans le cas présent.

Nous examinerons maintenant les avantages relatifs des deux conceptions montrées dans les figures 4 et 5 , des points de vae hydraulique et mécanique. 
lixa, 2.

Levage, en position de montage,

d"une rome de

turthine-pompe Dériaz,

a la centrale de

"Sir Adam Beck"

Niagara.
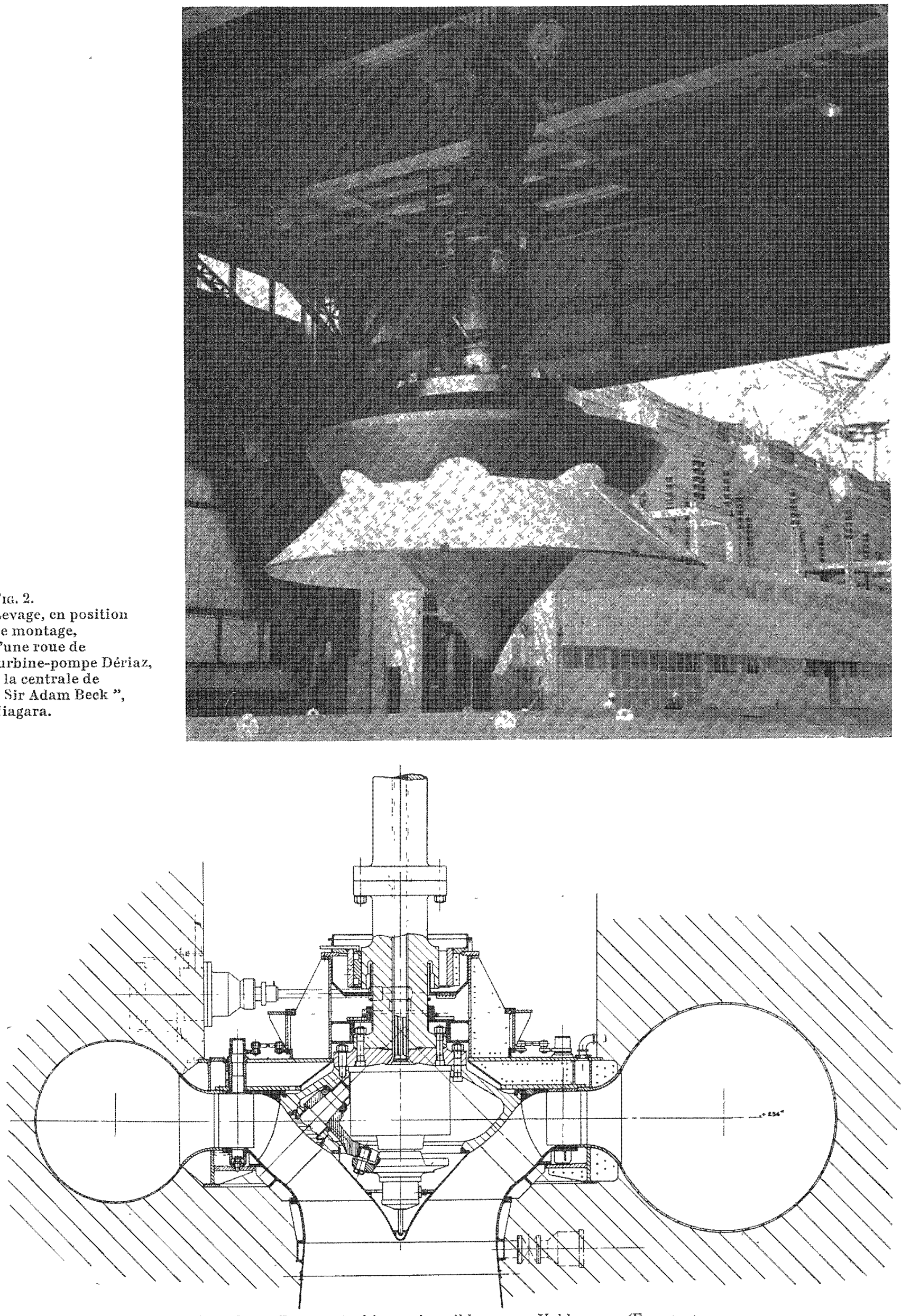

Fua, 3.-. Pompesutubines réversibles pour Valdecanas (Espagne).

Puissance de la turbine: $110000 \mathrm{B.HP}$ - Chute; $73 \mathrm{~m}$. - Puissance d'entré de la

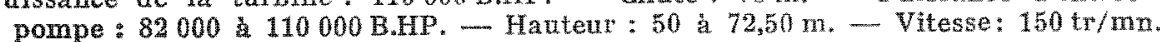




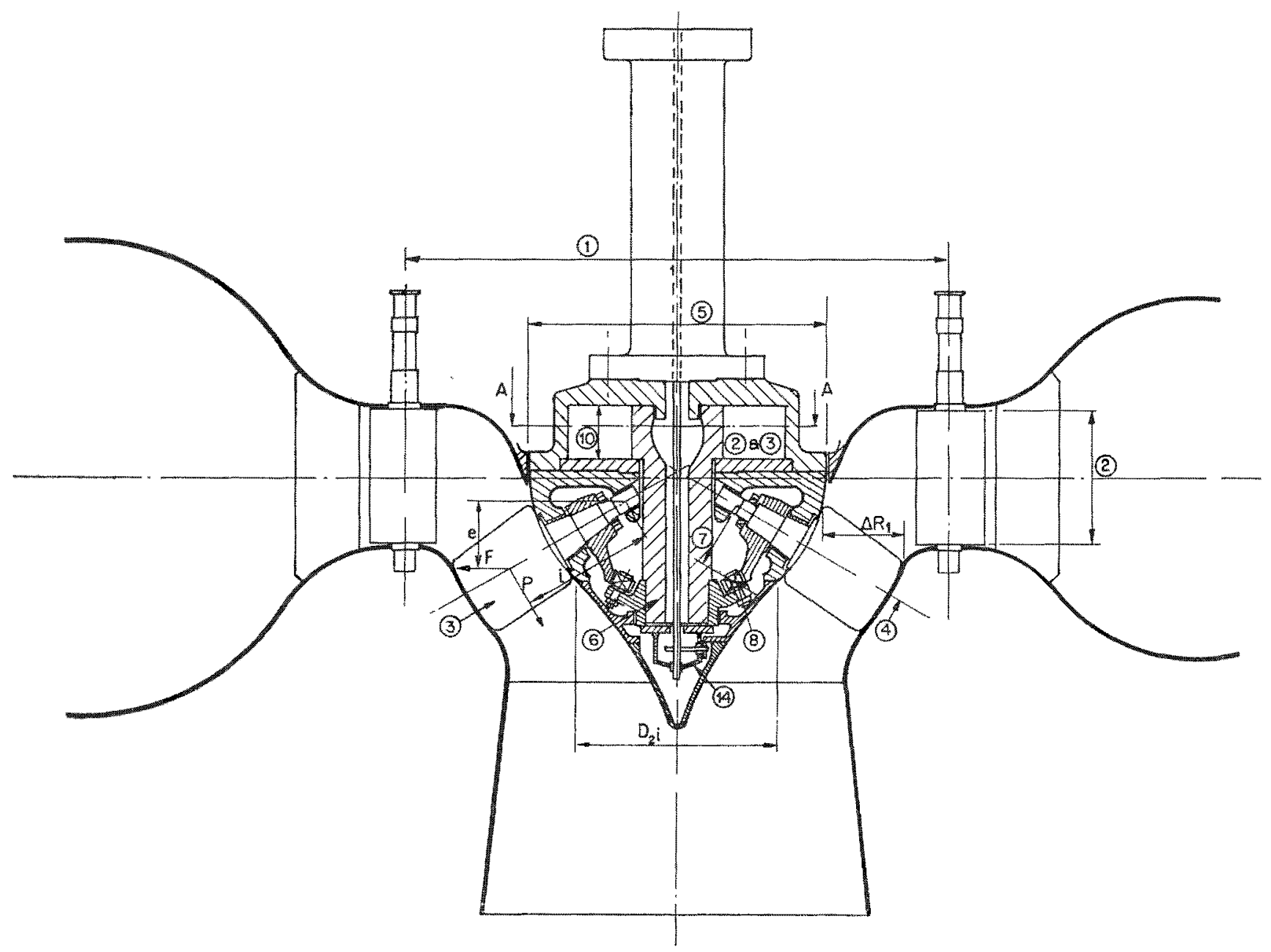

Fig. 4. - Turbine à écoulement mixte et pales orientables (Dériax). Puissance : 30000 B.HP. Chute : $55 \mathrm{~m}$ Vitesse : $300 \mathrm{tr} / \mathrm{mn}$

(1) Cercle de vannage, diamètre : $3,20 \mathrm{~m}$;

(2) Hauteur des directrices;

(3) Pales de la roue réglables autour de l'axe (4), qui est incliné par rapport à l'axe de l'arbre;

$\left(\Delta B_{1}\right)$ Etendue radiale du bord d'entrée de la pale:

$\left(\mathrm{D}_{\text {ii }}\right)$ Diametre du moveu a la sortie de la pale de roue;
(5) Joint hydraulique;

(6) Arbre de rotation du servomoteur;

(7) Levier de commande des pales;

(8) Glissière;

(10) Servomoteur à ailettes mobiles;

(14) Mécanisme d'asservissement du régulateur.

\section{CONSIDERATIONS HYDRAULIQUES}

$1^{\circ}$ En comparant les profils hydrauliques des figures 4 et 5 , on voit que celui de la figure 4 est plus «profilè », présentant un passage plus direct du vannage à l'aspirateur. Etant donné que le moyeu de la roue de la figure 4 est beaucoup plus court que celui de la figure 5 , le pérímètre mouillé se trouve diminué, avec une diminution correspondante des pertes par frottement.

$2^{\circ}$ La chute de $55 \mathrm{~m}$, élevée pour une machine
Kaplan, exige une vitesse spécifique relativement faible. L'étendue radiale de la pale $\Delta R_{1}$ devrait done être faible. Ceci est facilement réalisable sur la Dériaz sans augmenter les vitesses d'écoulement, car le rayon moyen à l'entrée des pales est plus grand. Dans le cas considéré, l'étendue radiale $\Delta \mathbb{R}_{1}$ de la pale de la Dériaz est de $8 \%$ plus petite que sur la Kaplan. On notera également qu'alors que les deux machines ont la même aire de sortie de la roue, l'aire d'entrée 


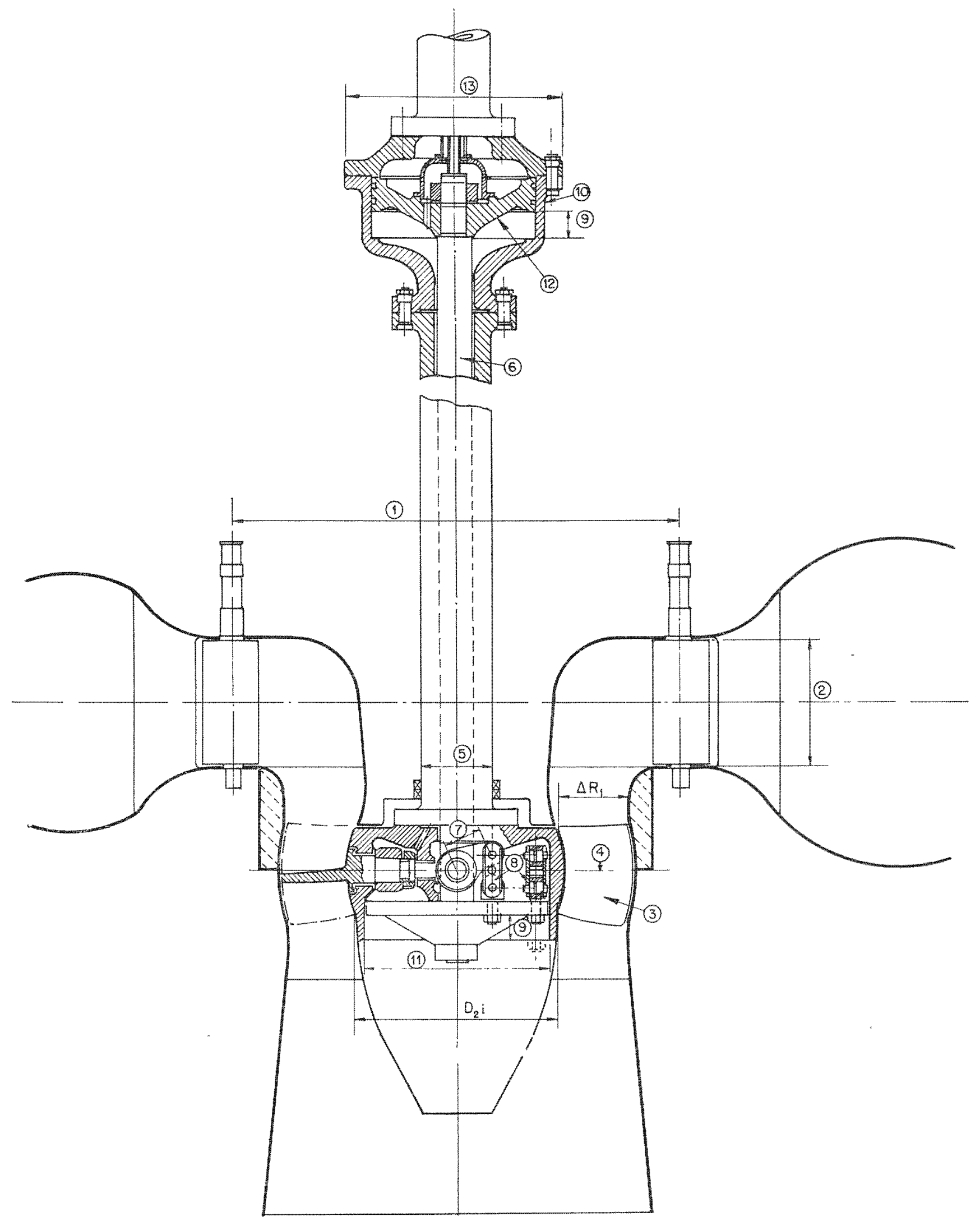

Fic. 5. - Turbine à écoulement axial et pales orientables (Kaplan). Puissance : 30000 B.HP. Chute : $55 \mathrm{~m}$ Vitesse : $300 \mathrm{t}, / \mathrm{mm}$

(1) Cercle de vannage, diamètre $3,29 \mathrm{~m}$;

(2) Hauteur des directrices:

(3) Pales de la roue réglables sulvant l'axe (4), qui est perpendiculaire à l'axe de l'arbre;

$\left(\Delta R_{1}\right)$ Etendue radiale du bord d'entrée de la pale;

$\left(D_{9 i}\right)$ Diamètre du moyeu a la sortie de la pale de roue;

(5) Diamètre du presse-étoupe de larbre;

(6) Tige de commande;
(7) Levier de commande des pales;

(8) Biellette double de commande des pales:

(9) Course du servomotem;

(10) Servomoten :

(11) Place disponible pour position en variante du serm vomoteur:

(12) Piston du servomoten:

(13) Diametre hors tout du servomoteur. 


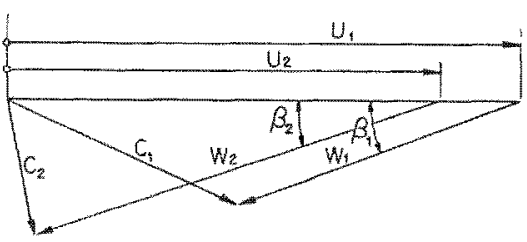

Fia. $6 a$

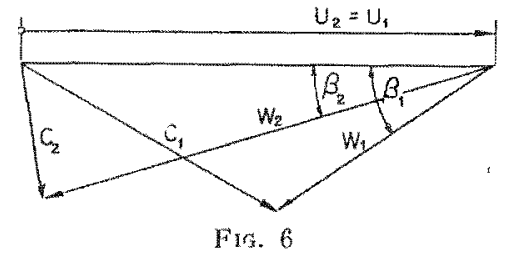

Fig. $6 \mathrm{~b}$

Diagrammes compares des vitesses pour les roues Deriaz (a) et Laplan (b) et pour no ligne d'econlement moyemne.

$u_{1}=$ vitesse périphérique de la rone, $\dot{a}$ l'entrée.

$u_{z}=$ vilesse périphérique de la roue, $\dot{a}$ la sortie.

$w_{1}=$ vitesse d'ecoulement relative à la rove, à l'entrée.

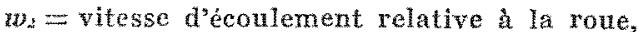
al la sortie.

$c_{1}=$ vilesse d'écoulement absolue, à l'antrée.

$c_{2}=$ vitesse d'ézonlement thosolue, $a$ la sortie.

de la Dériaz, par contre, est de $27 \%$ plus grande que sur la Kaplan. On obtient ainsi de faibles vitesses d'entrée, ce qui est favorable.

La figure 6 montre les triangles des vitesses d'entrée et de sortie pour le profil de pale moyen dans les machines Dériaz ef Kaplan.

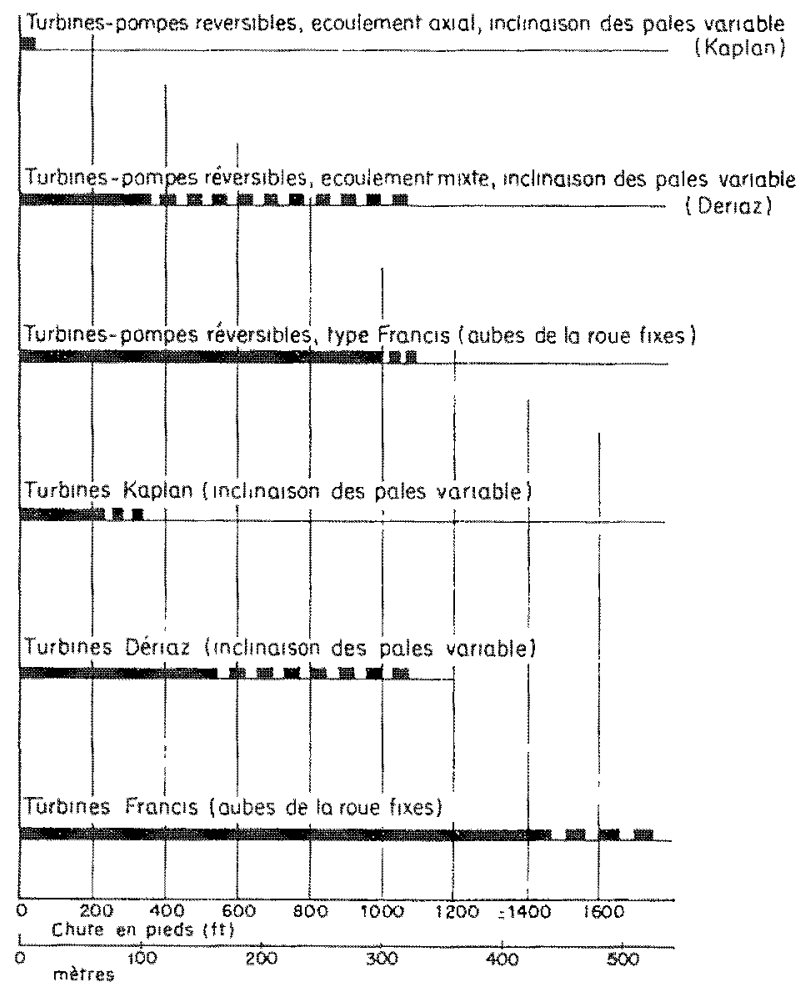

Etant donné la vitesse périphérique $a_{1}$ plus élevée de la Dériaz ef la vitesse d'écoulement d'entrée $c_{1}$ plus faible, les vecteurs de vitesse relative à l'entrée et à la sortie $w_{1}$ et $w_{2}$ sont presque parallèles, c'est-á-dire que les angles d'entrée et de sortie des pales $\beta_{1}$ et $\beta_{2}$ sont égaux. Sur la Kaplan, $w_{1}$ et $w_{2}$ sont divergents, comme on le voit d'après les triangles, et $\beta_{2}$ est plus petil que $\beta_{1}$. Lorsque la pale tourne, les angles d'entrée et de sortie des pales varient simultanément de la même quantité. Ces angles restent done parallèles sur la Dériaz, alors quils reslent divergents sur Ia Kaplan. Etant donné que les conditions d'écoulement exigent que $\beta_{1}$ et $\beta_{2}$ tendent vers zéro lorsque l'écoulement devient égal à zéro, la roue de la Dériaz reste en conformité avec les exigences hydrauliques, quel que soit l'angle des pales. Il en résulte che, aux très faibles charges, cette machine possède un rendement plus élevé que la Kaplan.

$3^{\circ}$ Le diamètre moyen à la sortie de la Dériaz est plus petit que sur la Kaplan, pour la même aire de sortie. Ceci résulte du plus faible diamètre de son moyeu à la sortie des pales $\left(\mathrm{D}_{2,3}\right.$, voir fig. 4 et 5). La vitesse périphérique $u_{2}$, et, par suite, la vitesse relative d'écoulement $w_{12}$ se trouvent ainsi réduites, ce qui améliore le coefficient de cavitation.

$4^{\circ}$ Le coefficient de cavitation se trouve égam

Fra, 7. - Chutes sous lesquelles peuvent fonctionner les pompes et turbines à simple étage. 
lement amélioré par le fait que les angles $\beta_{2}$ et $\beta_{2}$ sont éganx. Ceci veut dire que la zone de pression absolue minimale ne se trouve plus sur le côté convexe de la pale, comme sur la Kaplan (chose nécessaire à cause de la déviation de $\beta_{1}$ à $\beta_{2}$ ), mais qu'elle est située plus loin vers l'aval, à la sortie de la roue.

$5^{\circ}$ Aux vitesses anormalement élevées, la disposition à écoulement mixte tend à diminuer le débit. Ce phénomène est bien connu sur les turbines Francis à faible vitesse spécifique, et conduit à une vitesse d'emballement plus faible que sur la Kaplan, sur laquelle l'écoulement axial provoque des survitessés et des débits considérables en régime d'emballement. Grâce à cette réduction de la vitesse d'emballement, la génératrice correspondant à la machine à écoulement mixte revient à meilleur marché.

$6^{\circ}$ Grâce $\mathfrak{a}$ son diamelre de moyen plus grand à l'entrée des pales, la roue Dériaz produit une poussée hydraulique moins élevée que la Kaplan. Cecil est dû au grand diamètre du joint d'étanchêté (5) sur le moyeu (fig. 4) supérieur au faible diamètre du presse-étoupe de l'arbre de la Kaplan (fig. 5). L'espace entre le joint (5) et le pressemétoupe de l'arbre, sur la Dériaz, est en communication avec le canal de fuite.

$7^{\circ}$ Avec la chute relativement élevée que nous considérons, il est essentiel que le moyeu soit de forme sphérique afin d'éviter des jeux entre la pale et le moyeu pendant les rotations. Sur la Kaplan, ce moyeu sphérique provoque une nette modification de la direction des lignes d'écoulement dans la zone de basse pression, ce qui produit un risque de cavitation complémentaire. Grâce aux dimensions plus grandes du moyeu Dériaz, le rayon sphérique est plus grand et le changement de direction est moins net; de plus, ce changement a lieu bien à l'amont de la zone de basse pression, éliminant ainsi le risque de cavitation au noyeu.

$8^{\circ}$ Lors du fonctionnement en pompe, le modèle à écoulement mixte peut refouler à une hamteur beaucoup plus élevée que la machine à écoulement axial, comme on peut le voir à partir de la figure 7 . Cette figure a elé publiée dans l'article présenté par l'auteur au Congrès mondial de l'Energie (World Power Conference) de 1958 [3], mais elle a été retouchéc depuis lors en fonction des études récentes. Ces dernicres ont montré qu'il est possible d'étendre l'utilisation des machines à écoulement mixte à des chutes beancoup plus élevées qu'on ne l'avaif pensé au départ. Les zones pointillées de la figure 7 sont les zones d'extension de chute que l'on considere comme utilisables pour le fonctionnement lant en turbine qu'en pompage.

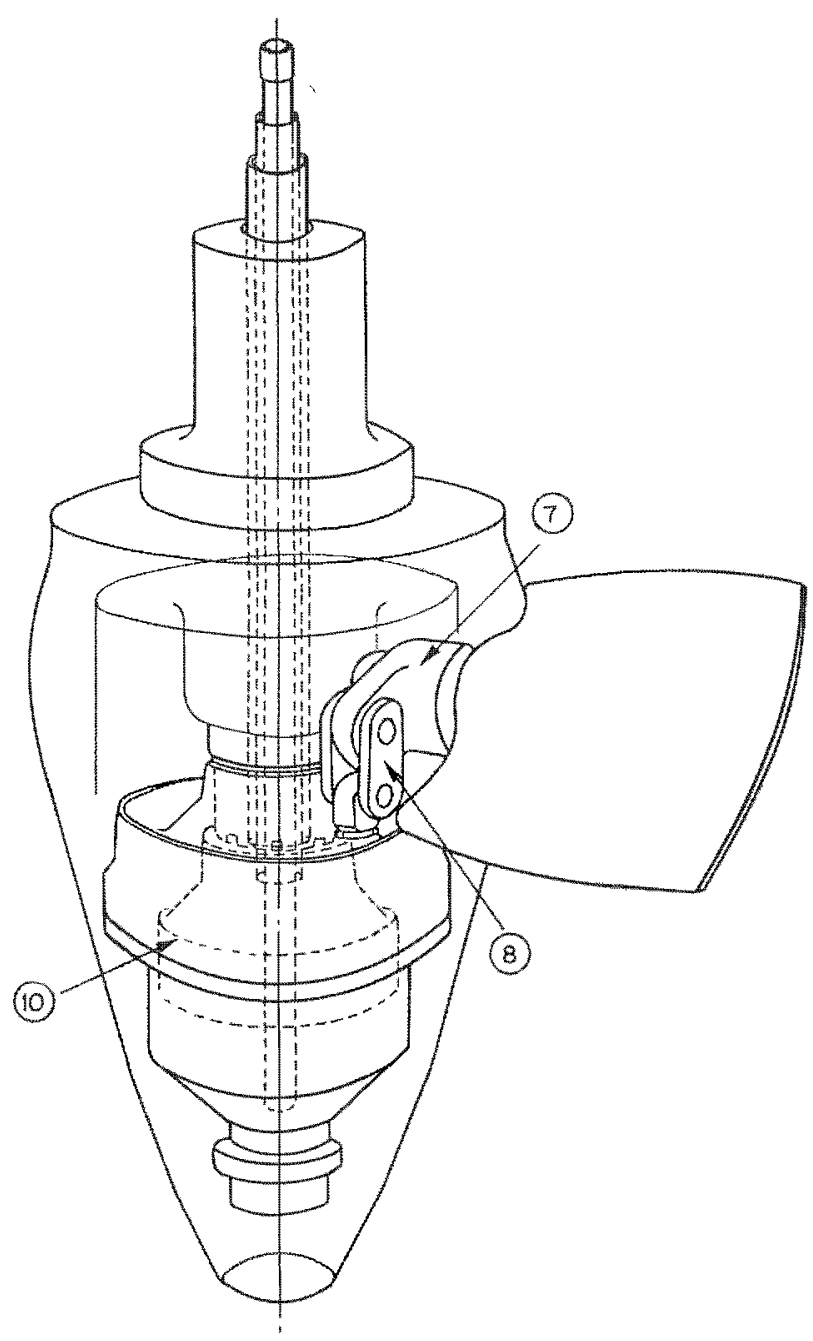

Fis. 8, - Vue perspective de la roue Kaplan.

\section{CONSIDERATIONS MECANIQUES}

1" Une chute importante nécessite un plus grand nombre de pales possédant des pivots relativement larges, de façon à pouvoir résister aux forces hydrauliques. Dans la présente étude, on a prévu 10 pales pour la Dériaz, alors que la Kaplan n'en comporte que 8. Ceci ne présente 


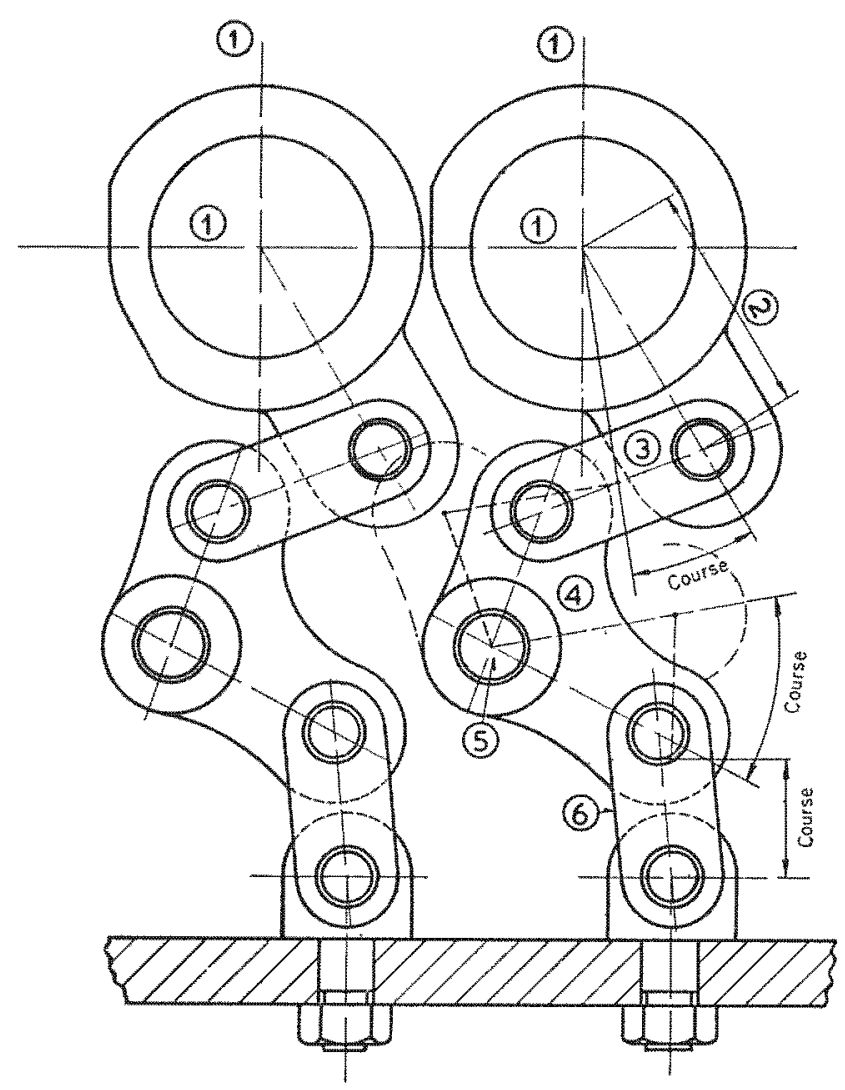

Fic. 9. - Timonnerie spéciale pour système Kaplan.

(1) Axes des tourillons des pales;

(2) Levier de commande;

(अ) Biellette double;

(4) Levier de renvoi a angle droit;

(5) Pivot fixe pour le levier (4);

(6) Biellette double.

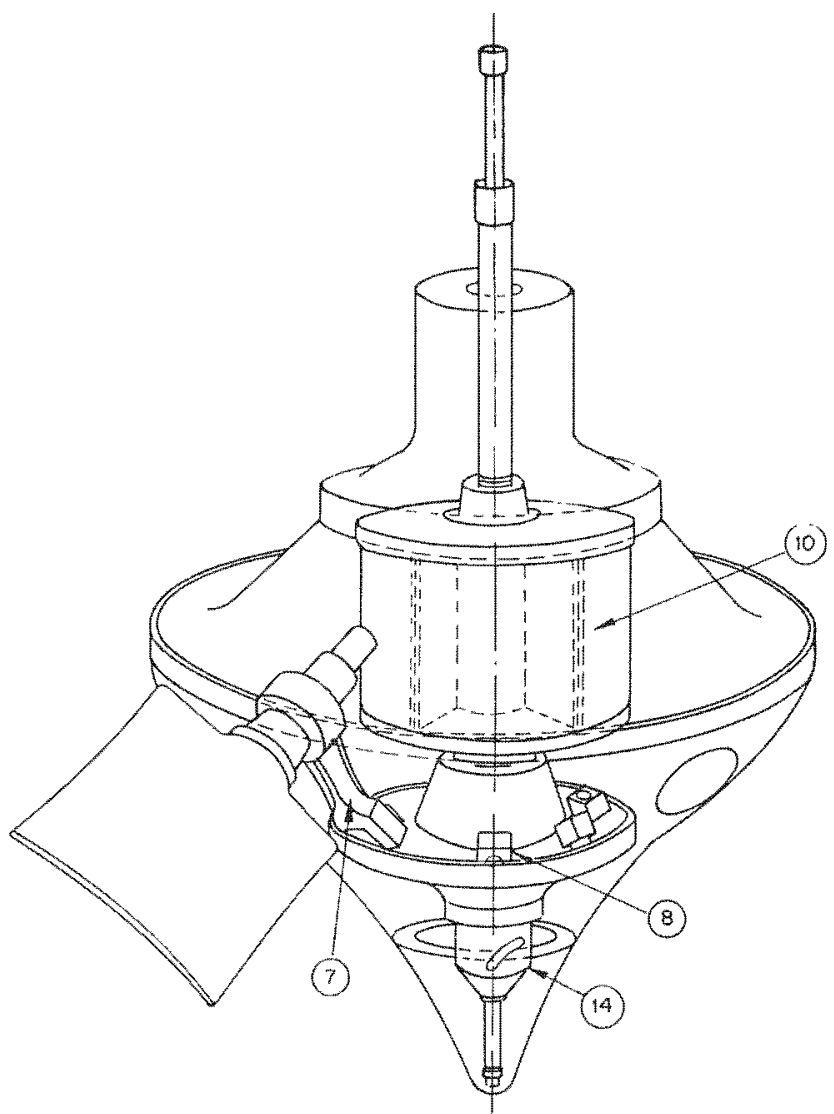

Fra. 10. - Vue perspective de la roue Déraz de la fig. 4 aucune difficulté, étant données les plus grandes dimensions du moyeu.

$2^{\circ}$ La position inclinée des pivots des pales fait que la force centrifuge $F$ provenant de la pale (voir fig. 4) produit un moment F.e qui s'oppose à celui de la pression hydraulique résultante P.i. Il s'ensuit que le plus grand palier de pivot, soumis à des charges importantes, est. particllement allégé. Dans la présente étude, cet allégement atteint $43 \%$ sur le plus grand des paliers et $13 \%$ sur le plus petit.

Dans la version Kaplan de la figure 5, il ne se produit aucune réduction correspondante des charges sur le palier, car la force centrifuge s'exerce selon le même axe que celui du pivot de la pale.

$3^{\circ}$ Pour les chutes élevées considérées, le mécanisme de commande classique des turbines Kaplan (voir fig. 5 et 8 ) devient très difficile à loger:

a) Il y a très peu de place disponible entre les gros paliers des pivots, et les leviers de commande (7) qui sont situés entre ces paliers deviennent extrêmement courts, à moins que l'on n'augmente le diamètre du moyeu beaucoup plus qu'il n'est à désirer du point de vue hydraulique.

b) Parce que le levier (7) est court, il en résulte des forces élevées sur les biellettes de liaison (8), et un raccourcissement des courses (9) de la tige de commande (6) et du piston du servomoteur.

La figure 9 montre une disposition employée parfois sur les Kaplan afin de réduire la limitation des dimensions du levier. Ici, le levier (2) est plus grand que ne le permettrait l'espacement des axes des pales (1). Il faut alors prévoir

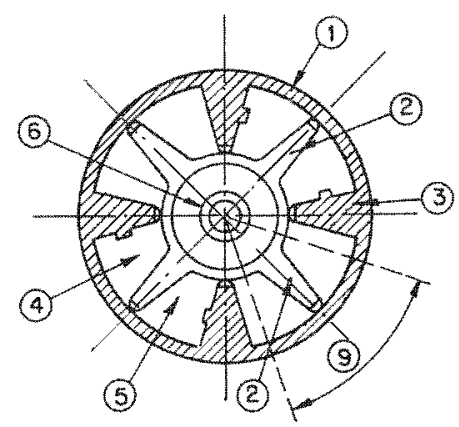

[PIa. 11

Servomoteur a alletteb. Coupe selon AA de la flgure 4.

(1) Corps du servomoteur;

(2) Allette mobile du rotor

(4) Chambre de ganche:

3) Allette fixe:

(5) Chambre de droite;

(6) Arbre de commande;

(9) Course de l'ailette (2) 


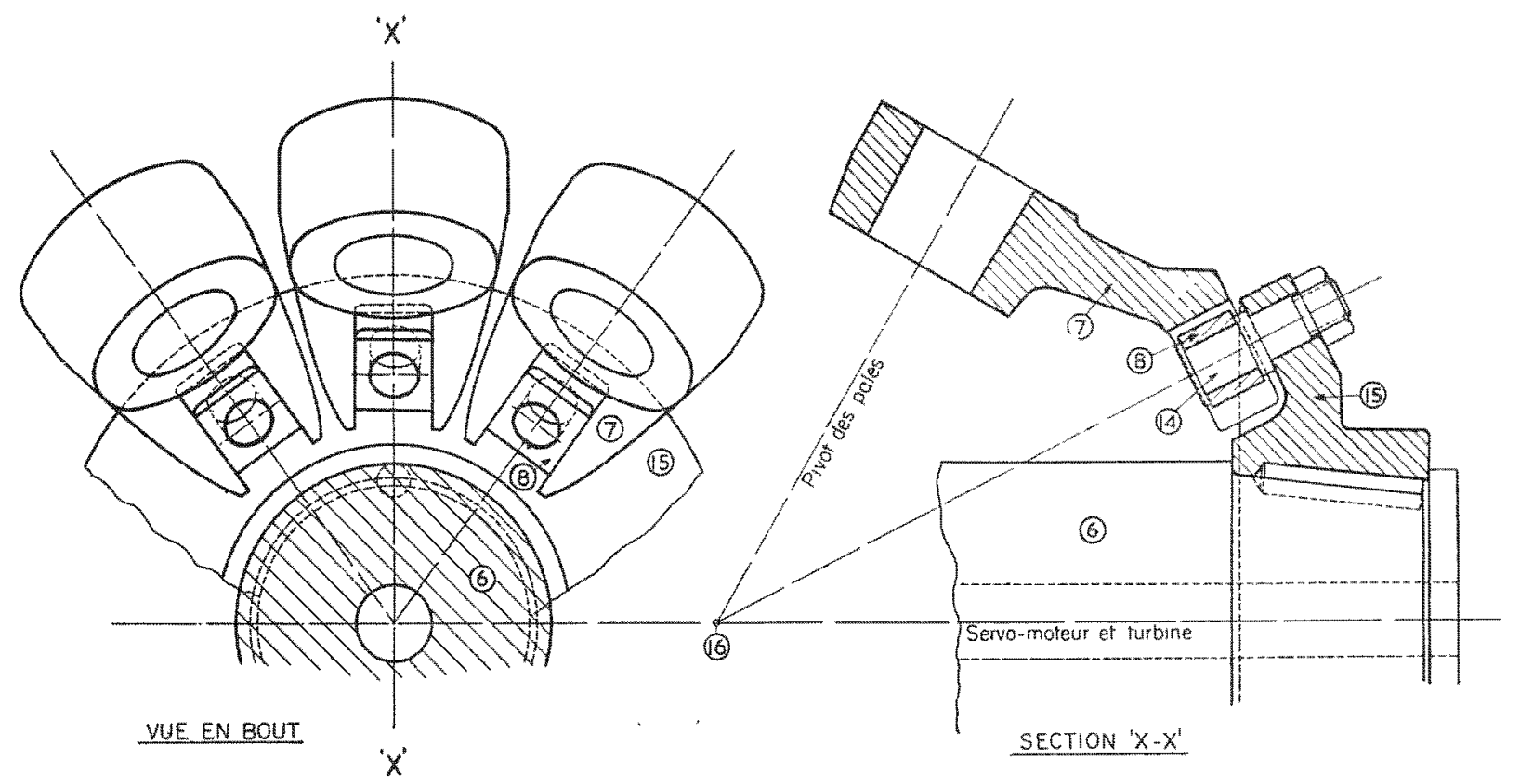

Fra. 12. - Mécanisme de transmission entre le servomoteur a ailettes et les tourillous des pales de la rone.

- (6) Arbre de rotation du servomoteur, coaxial avee laubre de la turbine;

(7) Levier de commande des pales de la rone;

(8) Glissière, pivotant sur (14);

(14) Axe monté sur le croisillon (1:3;

(15) Croisillon claveté sur (6).

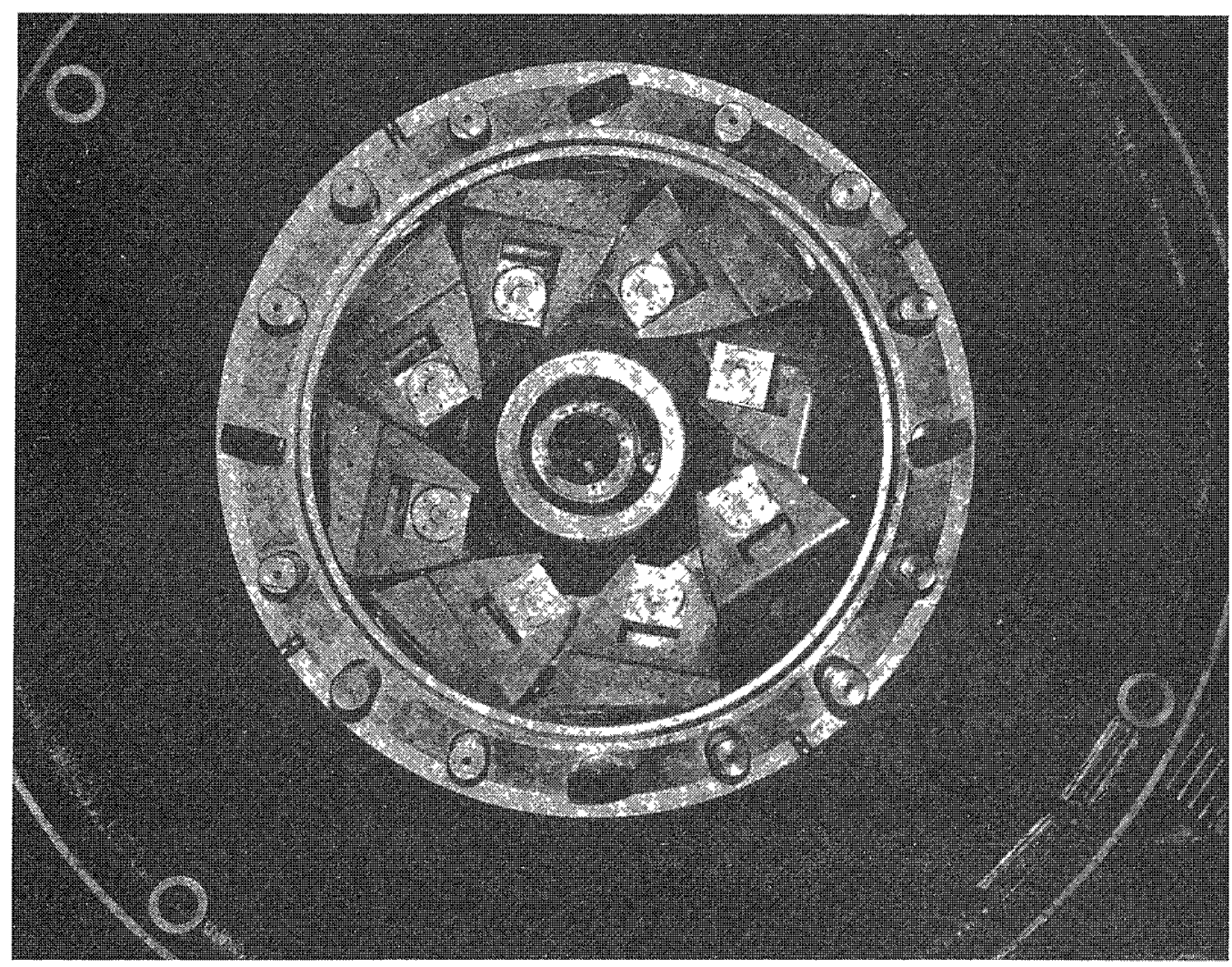

FIG. 13. - Vue du mécanisme des pales, à Niagara, montrant les extrémités bifurquées des leviers, avec les glissières lorsque les pales sont en position fermée. 
un levier de renvoi d'angle (4) ă pivot fixe (5) et deux biellettes doubles (3) et (6).

L'ensemble de ce mécanisme doit ètre logé dans l'espace réduit à l'intérieur du moyeu. Plus il y a d'axes et de paliers pouvant avoir du jeu, moins précis sera le réglage de position des pales de la roue. Compte tenu de la bonne finition el de la précision des profils, nécessaires pour les pales Kaplan, l'introduction de dispositifs permotlant éventuellement une inégalilé de positîns angulaires des pales semblerait être à déconseiller.

Ces difficultés sont éliminées de la manière suivante sur la Dériaz, représentée par les figures 4 et 10 :

a) A mi-course, les leviers (7) sont dans le plan radial des pirots des pales, ce qui permel ainsi de les loger sans difficulté. Ils peuvent donc être plus longs, malgré le fait que les pales sont plus nombreuses.

b) Il n'y a pas de bielletles hu genre (8) des figures 5 et 8 . La transmission s'effectue directement par la glissicre (8) des figures 4 el 10 .

$4^{*}$ Lo servomoteur des pales de la roue est l'élément le plus différent sur les Dériaz et sur les Kaplan (voir fig. 4 el 5). Sur la Kaplan, la course (9) du servomoteur est axiale par rapport à l'arbre de turbine. On peut normalement disposer le servomoteur sur l'arbre au point (10) où aucune réduction de diamètre n'existe.

Sur beaucoup de machines Kaplan, le servomoteur peut être situé à la position montrẻe par (10) sur la fig. 8 et repérée par (11) sur la fig. 5 , c'est-àdire à l'intérieur du moyeu, ce qui evite la longue et lourde tige de piston (6). Ceci n'est pas possible pour les hautes chutes, puisque le diamelre (13) est plus grand que l'espace disponible (11). Une manière de résoudre cette difficulté est d'augmenter la pression de l'huile du régulaleur afin de permettre de réduire le déplacement volumétrique du servomoteur; cette pression peut atteindre $28 \mathrm{~kg} / \mathrm{cm}^{2}$, mais ceci n'est pas courant. Une autre méthode consiste à installer un servomoteur double « en tandem » dans l'espace au-dessous du diamétre (11) dans le moyeu, ce qui est un travall très compliqué.

La figure 4 montre la machine Dériaz avec un scrvomoteur (10) à ailetles mobiles. La figure 11 montre la coupe A.A. de cette disposition, où l'on voit 4 ailetles fixes (3) attachées au corps (1) et 4 allettes mobiles (2) montées sur larbre de commande $(6)$. Ces ailettes délimitent 8 compar. timents, dont 4 (à gauche) produisent un mouvement dans un sens lorsqu'on les remplit, alors que les 4 autres (à droite) communiquent avec l'évacuation, et vice-versa.

La hateur du servomoteur (10) (voir fig. 4), peut être variée pour s'adapter à l'énergie néces- saire à la commande des pales de la roue sans augmentation du diamètre global du corps du servomoteur. La course angulaire (9) de l'arbre (6) se voit d'après la figure 11. Son déplacement est transmis aux leviers (7) de la figure 10 par un mécanisme dont le détail est présenté dans la figure 12. Afin de rendre la présentation plus claire, on a omis le pivot de la pale, n'indiquant que son axe. De même, on a omis les axes (14) dans la vue en bout.

Le mécanisme est montré en position «micourse $\gg$, comme dans la figure 11 . A mesure que l'arbre du servomoteur (6) change de position, les leviers (7) s'inclinent, comme on peut le voir sur la photographie de la figure 13, dans laquelle les leviers sont en position «pales fermées».

La condition géométrique poux un fonctionnement correct est: l'axe médian de l'axe (14) et de sa glissiere passe par le point (16) où laxe du pivot des pales recoupe l'axe du servomoteur. Cette disposition est nouvelle et fait l'objet d'un brevet.

La fabrication du servomoleur à ailelles de la figure 11 est un peu plus complexe aue cello d'un servomoleur cylindrique ordinaire, dont le piston se déplace dans le sens axial. II en résulte cependant une simplificalion considérable à d'autres points de vue, ce qui le justifie enticremenl du point de vue ćconomique. Ce dispositif s'esl montré complètement sûr en fonctionnement.

$5^{\circ}$ Lorsqu'on etudie une turbine Kaplan pour des hautes chutes, il est essentiel que l'enveloppe de la roue soil sphérique, tant au-dessus $q u^{\prime}$ au-dessous de l'axe de la pale, afin qu'll $\mathrm{n}^{\prime} \mathrm{y}$ ait qu'un faible jeu entre la pale et l'enveloppe. II s'ensuil la nécessité de prévoir une partie supérieure amovible de l'enveloppe afin de permettre le démontage de la machine. La partie ombrée de la figure 5 indique la partie de lenveloppe qui doit être amovible. Avec la roue Dériaz, cette difficulté se trouve éliminẻe. La surface sphérique se prolonge tout le long du bord extérieur des pales et cette pièce peut être scellée dans le béton une fois pour toutes puisqu'il n'est jamais nécessaire de la démonter par la suite.

$6^{\circ}$ La cavitation provoquée dans l'espace entre les extremités des pales et l'enveloppe nécessite une attention toule particulière, surtout pour les hautes chutes. Pour une roue axiale (Kaplan), ce jeu est fixé lors de la fabrication et ne peut être varié par la suite à moins que lon ne modifie les extrémites des pales.

Sur la machine à écoulement mixte (Dériaz), ce jeu est facilement réglable en calant la roue de telle manière que seul le jeu requis pour le fonctionnement subsiste. Ce réglage ne nécessite aucun démontage; il s'effectue en ajustant les semelles du palier de butée de l'arbre. 
Il s'ensuit done que la disposition à écoulement mixte convient beaucoup mieux aux chutes élexées que la disposition à écoulement axial.

7" Grâce au moyeu de roue plus court, et à la génératrice plus légère, on peut installer mn pont roulant plus bas et de puissance moins élevée avec la machine Dériaz, ce qui nccasionne une très appréciable économie de frais de cons. truction.

\section{RESULTATS EXPERIMENTAUX}

Une étude comparative complete du fonctionnement des turbines Kaplan et Dériaz a été effectuée à Ia station d'essais du Service hydraulique de $l^{\prime}$ "English Electric Company », à Rugby, en vue d'examiner l'utilisation d'une turbine Dériaz pour une commande recue du * North of Scotland Hydro-Electric Board $\%$. Nous venons de recevoir les résultats de ces essais el nous les présentons sommairement ci-dessous. coude d'aspiratcur étaient les mêmes pour les deux essais.

La figure 14 montre la poue (Dériaz) du modèle d'essai avec le mécanisme de vannage classique, dans la station d'essais.

Ces résultats donnent lieu aux remarques suivantes:

1) La vitesse optimale de la roue Dériaz est

RÉSUME DES ESSAIS SUR MODELE DE TURBINES

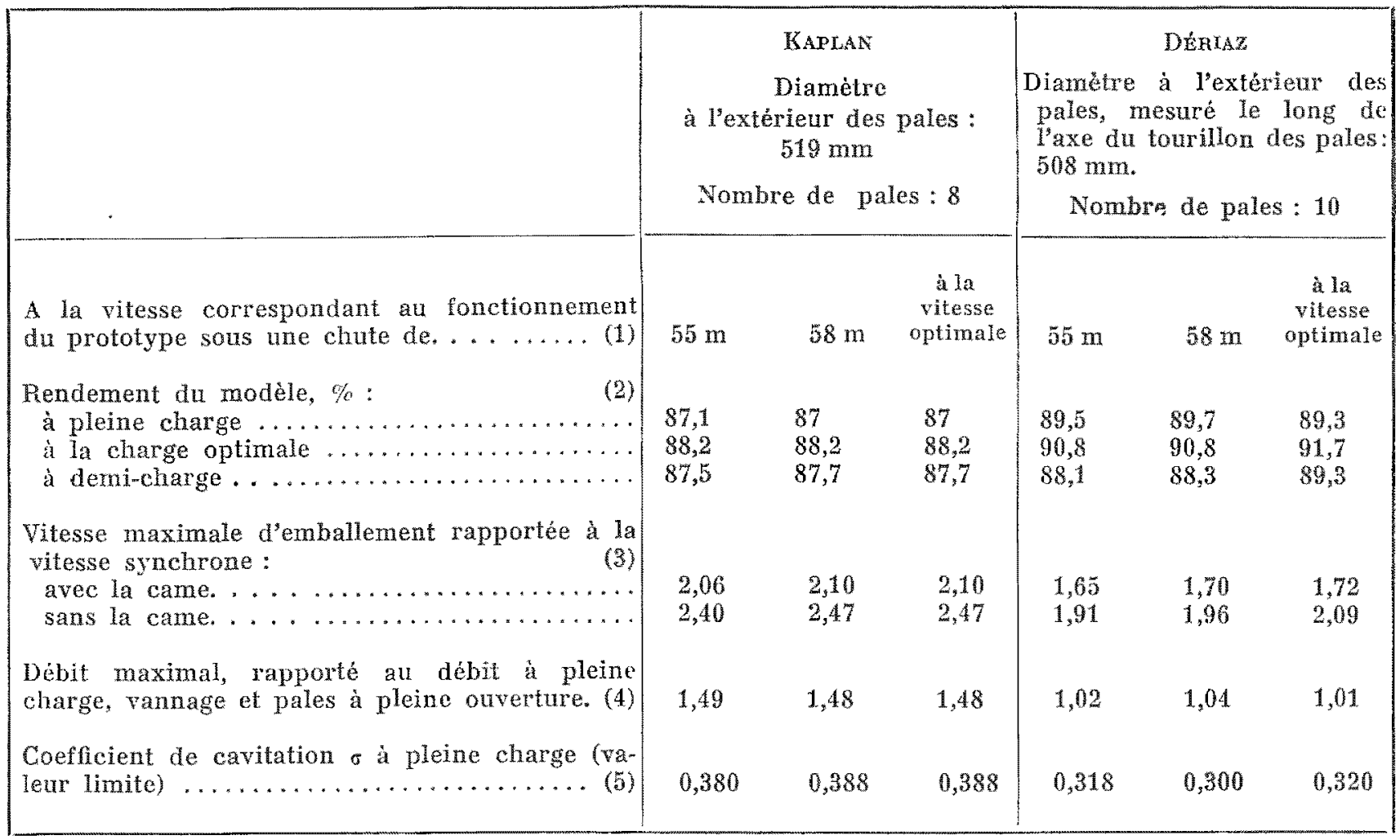

Les essais des rones modèles Kaplan et Dériaz ont été effectués sur le même bane d'essais avec les mêmes étalonnages. A part la roue et la ceinture de roue, les seules différences entre les deux bancs d'essais concernaient la partie supérieure de l'aspirateur et le plafond. La bâche spirale, le mécanisme de vannage et le légèrement inférieure ţ celle de la roue Kaplan;

2) Les rendements sur modele sont ceux obtenus sur un banc d'essais sans effet d'échelle.

On notera que les rendements obtenus avec la turbine Kaplan étaient relativement faibles. Nous obtenons normalement 90 et $91 \%$ sur nos 
modeles de turbines Kaplan pour des vilesses spécifiques de 400 à $600 \mathrm{~N}_{3}$ (en unités métriques). Pour la chule très élevée considérée, les rendements sont abaissés de 2 ou 3 points en raison de la dimension excessive de la roue Kaplan, qui conduit à un profil hydraulique défavorable (voir les paragraphes 1 el 2 de l'arlicle).

3) La vilesse d'omballement très réduile de la roue Dériaz a permis une modification profonde de la conception de l'alternateur, ce qui, à son tour, a permis d'utiliser un pont roulant plus léger.

4) Les débits maximums indiqués au tablean sonl les maximums absolus pour n'importe quelle survitesse, y compris la vitesse d'emballement.
Ce débil maximum détermine le volume d'exca vation nécessaire pour la longue galerie de fuite. L'avantage de cette réduction du débil maximum est donc très important du point de vue du génie civil.

5) Le coefficient de cavitalion o (valeur limite) est la valeur la plus basse a laquelle le fonctionnement est encore satisfaisant.

Ces résultats d'essais étayent l'opinion exprimée au début de cet article, c'est-à-dire que, pour des chutes dépassant $36 \mathrm{~m}$, la turbine à écoulement mixle possède de nets avantages, et devrait remplacer la turbine purement axiale.

A la suite de ces essais, il a été décidé de procéder à la fabrication de la turbine Dériaz, d'une puissance nominale de 30500 B.HP. sous une

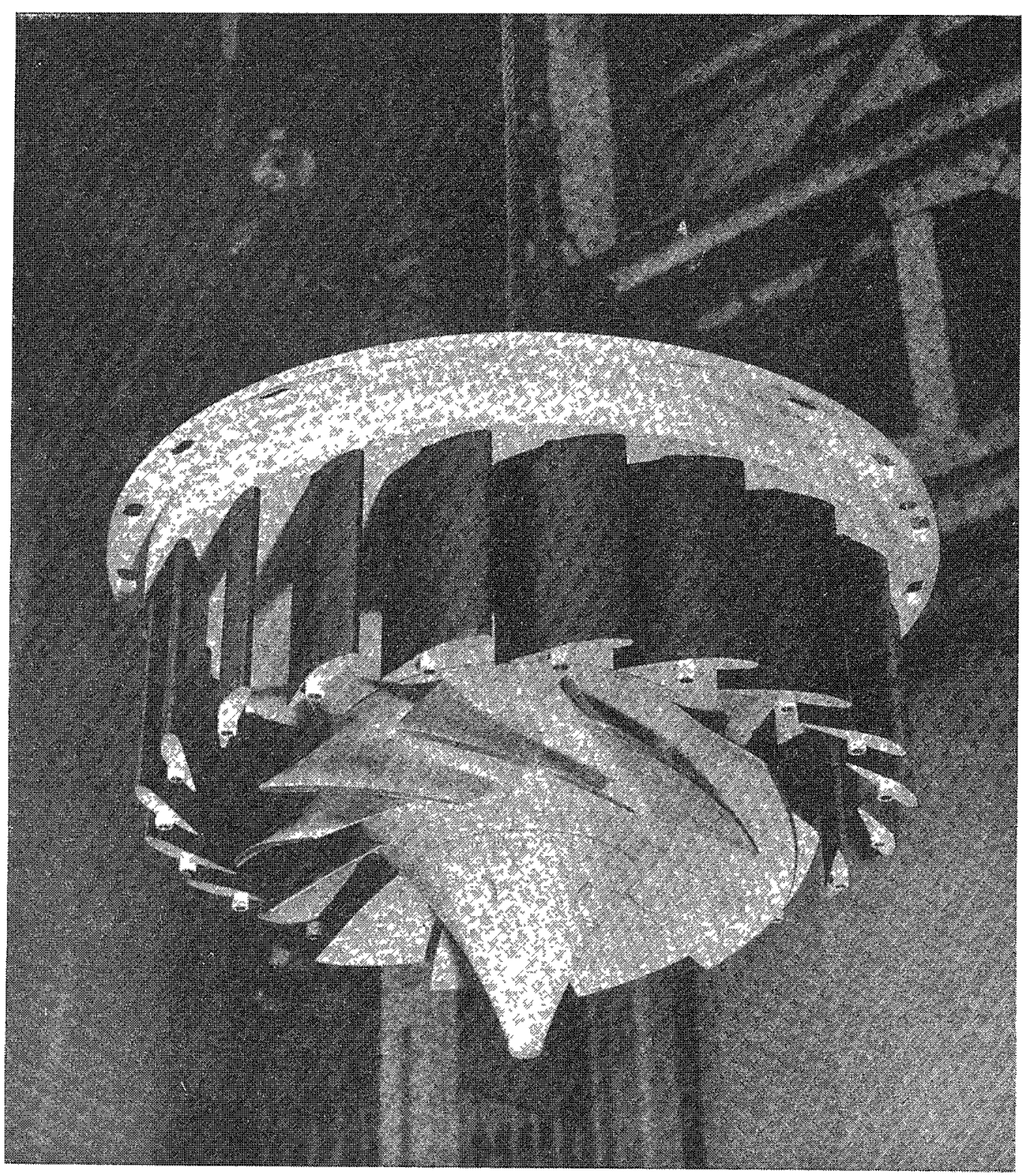

Fia. 14. - Nodele dessai de turbine pour la centrale de Culligran. 
chute de $55 \mathrm{~m}$ et de 32500 B.HP. sous une chute de $58 \mathrm{~m}$, à la vilesse de $300 \mathrm{tr} / \mathrm{mn}$. La turbine et la génératrice seront étudiées et fabriquées par 1' English Electric Company», et installées à la centrale de Culligran, du « North of Scot- land Hydro-Electric Board $»$, dont les ingénieursconseils sont MM. Kennedy et Donkin, de Londres. La fabrication de cette machine est actuellement parvenue à un stade avancé.

\section{CONCLUSIONS}

Nous avons, dans le prósent article, indiqué les avantages de la disposition à écoulement mixte pour une roue à pales réglables. L'auteur est d'avis que les utilisations de cetle disposition augmenteront dans l'avenir. Les possibilités d'utilisation de ce systeme n'ont pas encore été entièrement explorées. Surtout dans le domaine des groupes réversibles (turbinepompe) utilisés pour l'accumulation d'énergie par des moyens hydrauliques, la machine Dériaz à écoulement mixte est la sevle solution comportant une pompe centrifuge dont la puissance d'entrée soit réglable sur des gammes étendues avec de bons rondements. Ceci est essentiel pour des hautes chutes el des groupes de dimensions très importantes. Cette considération permet une grande souplesse de fonctionnement du cycle de pompage, quí devient comparable à celle du cycle de fonctionnement en turbine. C'est précisément cette souplesse qui est à la base de la très grande appréciation de la turbine hydraulique en tant que moteur de génération pour des charges de pointe. Cette même souplesse est maintenanl disponible lors du cycle de pompage.

\section{REMERCIEMENTS}

L'auteur remercie l'English Electric $C^{\circ}$ Ltd, qui lui a permis de présenter la matière de son article, ainsi que l'Indian Institute of Science pour lui aroir permis de le reproduire dans cette revue.

\section{REFERENCES}

1] P. Denaza - La tumbine pompe reversible axio-centrifuge à pas variable.

Bulletin technique de la Suisse Romunde, 20 ootobre 1.955.

2 P. Drnisz and J,G. WAnNock. - Revershle pumpfurbines for Sir Adam Beek, Niagara, Pumping Generating Station.

A.S.M.E., Transactions, Series D, Journal of Basic Engineering, December 1959.
3. P. Démz and J. G. Warnock .... Economic advantage, of variable-pitch rumers for water turbinepumps.

Wrold Power Conference, Paper $130 \mathrm{~A}_{4}, 3$, Montréal, September 1958.

[4] P. Dornz. - The mixed-flow rariable-pitch pumpturbine.

Water Poner, February 1960. 